Running Head: CREATIVITY AND PERSONALITY

\title{
Creative Ability, Creative Ideation and Latent Classes of Creative Achievement: What is the Role of Personality?
}

\author{
Sophie von Stumm ${ }^{\mathrm{a}^{*}}$, Audrey Chung ${ }^{\mathrm{b}}, \&$ Adrian Furnham ${ }^{\mathrm{b}}$ \\ ${ }^{a}$ Department of Psychology, Goldsmiths University of London, New Cross, SE14 \\ 6NW, London, UK. \\ ${ }^{\mathrm{b}}$ Department of Psychology, University College London, 26 Bedford Way, WC1H 0AP, \\ London, UK.
}

Key words: Creative Ideation; Divergent Thinking; Creative Achievement; Latent Class Analysis; Personality.

*Corresponding author:

Sophie von Stumm

Department of Psychology

Goldsmiths University of London

New Cross

SE14 6NW

London, UK

Tel.: +44-20-7979 7171-4286.

E-mail address: psp01svs@gold.ac.uk (Sophie von Stumm). 


\begin{abstract}
Silvia and colleagues (2009) highlighted the advantages of latent class analysis when studying creative achievement [Silvia, P. J., Kaufman, J. C., \& Pretz, J. E. (2009). Is creativity domain-specific? Latent class models of creative accomplishments and creative self-descriptions. Psychology of Aesthetics, Creativity and the Arts, 3, 139-148]. The current study replicates and expands Silvia et al.'s (2009) findings on creative achievement in a sample of 656 students, of which 223 were also assessed on creative ability and ideation, as well as a broad range of personality traits.

A latent class analysis identified three groups of non-achievers, average achievers with high interpersonal competence, and high creative achievers; the adequacy of this class solution was further supported by mean differences in divergent thinking, creative ideation and personality in the expected directions. In a multinomial regression model, hypomania was identified as consistent, significant predictor of creative achievement class membership. Overall, creative ability, ideation and achievement were shown to be only loosely inter-related, complicating the evaluation of personality's role in creative competence.
\end{abstract}


Madness is to think of too many things in succession too fast, or of one thing too exclusively. Voltaire (1694-1778)

There is no unequivocal definition or clear measurement of creativity, but a number of theoretical concepts and methodological approaches are scattered across the literature (Batey \& Furnham, 2006; Prentky, 2001; Taylor, 1988). This confusion has led to multifarious assessment instruments of creativity and corresponding debates about its operationalization as trait versus ability and its domain generality versus domain specification (e.g. Carroll, 1993; Feist, 1998; Guilford, 1950; Silvia, Kaufman, \& Pretz, 2009). In this study, we propose that creativity is a competence that results from three core elements of creative ability or capacity, creative ideation or thinking disposition, and creative achievement and accomplishment. Therefore, we aim to a) explore the interrelations of creative ability, ideation and accomplishment, b) investigate domainspecification versus domain-generality in creative achievements and c) evaluate the role of personality traits in creative competency.

\section{Creative Competence}

Previous research studies often relied on divergent thinking tests to conceptualize creativity (e.g. Furnham, Batey, Anand, \& Manfield, 2008; Silvia et al., 2008; Silvia, Nusbaum, Berg, Martin, \& O’Connor, 2009), which are commonly interpreted in terms of 
fluency and originality (Batey \& Furnham, 2006; Runco, 1991), and thought to indicate creative potential or the ability to generate original ideas (Silvia et al., 2008). Divergent thinking is positively related to general intelligence, specifically to verbal ability (e.g. Carroll, 1993; Hargreaves, 1927), and is necessary but not sufficient for creativity (Guilford, 1950). In line, divergent thinking scores are treated here as marker of creative power or an individual's maximum creative performance. Traditionally, psychometric researchers have distinguished between maximum performance, which refers to what an individual can do, and typical performance that infers what an individual's behavioral tendency or what he or she will do (Fiske \& Butler, 1963). In this context, we propose creative ideation as an indicator of typical creative performance, complimenting one's ability of idea generation. Runco, Plucker and Lim (2001) developed the Runco Ideational Behaviour Scale (RIBS) and defined creative ideation in terms of actual overt behaviors that reflect "the individual's use of, appreciation of and skill with ideas" (p. 394). The scale comprises 23 statements of ideational behaviors that assess individual differences in developing and managing ideas, such as the typical frequency of generating ideas and one's level of absorption with a particular thought (Runco et al., 2001). One earlier study indicated that creative ability or divergent thinking was marginally associated with creative ideation (Plucker, Runco, \& Lim, 2006), confirming previous findings on the modest overlap of maximum and typical performance measures (Ackerman, 1996). Therefore, low inter-relations of creative ability and ideation may not be readily interpreted in terms of nomological independence but are likely to comprise conceptually related pillars of creative competence. Theoretically, creative ability and 
ideation should be associated with creative achievement, which constitutes the third component of creative competence.

Carson and colleagues (2005, p.37) defined creative achievement as "the sum of creative products generated by an individual in the course of his or her lifetime". Creative achievement has been typically measured in terms of markers of eminence (e.g. Simonton, 1976), subject-matter expert ratings of creative products (e.g. MacKinnon, 1962) and self-report scales of everyday creative achievement (e.g. Batey, 2007). In addition to this variety of measurements, researchers have (and continue to) debated if creative achievement was be domain-specific or domain-generalist (e.g. Carson et al., 2005; Silvia et al., 2009). The domain-specific perspective suggests that individuals achieve only in selected domains of creativity; that is, one is either a painter or an inventor but rarely both. In contrast, the domain-general approach proposes that highly creative people will accomplish across several domains and therefore, perform similarly well poetry, ballet and clarinet. Most previous researchers attempted to settle this argument using multivariate statistics, especially exploratory and confirmatory factor analysis. In this context, Silvia and colleagues (2009) recently pointed out that multivariate latent trait models were unsuitable to solve the issue but that latent class analysis was more appropriate. Specifically, multivariate models assume that samples are homogenous and that people vary in amount but not kind. If creative achievement was domain-specific, however, samples must be heterogeneous violating the multivariate assumptions and hence, distorting the results.

Silvia et al. (2009) analyzed data from the creativity achievement questionnaire (CAQ; Carlson et al., 2005), which measures creative accomplishments in ten domains 
with additively weighted items that are scaled from lower to greater creative accomplishments. Silvia et al. (2009) identified three classes of creative achievement, including visual arts, performing arts and a non-creative class. In addition, the authors applied latent class analysis to self-descriptions of creativity, and concluded that classes represented quantitative, ordered differences rather than exclusive qualitative groups. Conversely, Batey and Furnham (2006) stated that operationalizations of creativity as abilities (i.e. divergent thinking) or behavioral tendencies (i.e. ideation) constitute normally distributed trait dimensions, whereas the distribution of creative achievement tends to be skewed.

\section{Personality and Creativity}

In differential psychology, creativity has been traditionally examined with reference to intelligence and personality (e.g. Barron \& Harrington, 1981). Effects of intelligence were modest or even trivial (e.g. Batey \& Furnham, 2008; Furnham et al., 2008); personality, however, has been repeatedly shown to account for substantial amounts of variance in creativity (e.g. Feist, 1998; Furnham et al., 2008). Eysenck (1993) argued that Psychoticism from the Gigantic Three was the core basis for creative thinking but this association is to date not conclusively proven (Batey \& Furnham, 2006). To explore associations of creativity and psychoticism further, a recent series of studies focused on the related traits of schizotypy and hypomania (e.g. Batey \& Furnham, 2009; Claridge \& McDonald, 2009; Furnham et al., 2008; Rawlings \& Georgiou, 2004). Both constructs evaluate an individual's proneness to psychosis but emphasize different 
pathologies and symptoms. Schizotypy comprises a tendency for unusual experiences, such as hallucinations, introvertive ahedonia, indicating ambivalence and inappropriate affect, cognitive disorganization, implying concentration difficulties, and impulsive nonconformity, which refers to the anti-social, aggressive side of psychosis (Claridge et al., 1996). Creative people show a high tolerance for ambiguity, are often deeply absorbed in thoughts and hence, may appear absent-minded or even disorganized, and withdraw themselves from social events (Sternberg \& Lubart, 1991). Hypomania is a primary feature of bipolar disorder and is characterized by irritability, racing thoughts, illusions of grandeur, risk taking and decreased need of sleep (DSM-IV-TR, 2000). Creative individuals are characterized by a high or even exuberant frequency of idea generation, a strong sense of self-belief and self-confidence, and ambitious determination and persistence in their creative pursuits (Furnham et al., 2008). Overall, moderate levels of schizotypy and hypomania are conceivably conducive for creative thought and accomplishment. Beyond the realm of psychosis-related traits, Openness to Experience from the Big Five has been repeatedly found to positively relate to creativity across domains and measurements (e.g. Feist, 1998; Feist \& Barron, 2003; Silvia et al., 2008; 2009). Openness comprises facet scales of fantasy, imagination and ideas, all of which tap core characteristics of creative individuals. For other FFA traits, including Neuroticism, Extraversion, Agreeableness and Conscientiousness, the evidence is currently less conclusive (Batey \& Furnham, 2006; Feist, 1998).

\section{This Study}


Silvia et al's (2009) identification of three latent classes of creative achievers has to date not been replicated. Furthermore, Silvia et al. (2009) examined classes of creative achievement with reference to only two personality traits of Openness to experience and Extraversion, as well as studying arts at university. In the current study, we aim to replicate the latent class solution of creative achievement using the biographical inventory of creative behaviors, which measures spontaneous everyday creativity (Batey, 2007). If a fitted latent class model included distinct latent classes, domain-specification of creativity is likely. If the analysis resulted in a one-group model or simple quantitative class profiles, creative achievement is likely to be domain-general. Furthermore, we will investigate classes of creative achievers with respect to creative ability and ideation to advance the understanding of creative competence. Finally, we hope to identify those personality traits from a wide range of broad and narrow constructs, which are consistently associated to components of creative competence.

\section{Methods}

\section{Participants}

This study included an overall sample of $N=656$ participants with 222 males and 434 females. The total sample comprised several groups of university students across the United Kingdom, whose data were aggregated from several research projects. Overall, 651 participants reported their age ranging from 13 to 58 years with a mean of 20.53 
years $(S D=5.94)$. For a sub-sample of 223 (139 males and 84 females $)$ participants, additional data on personality and creativity were collected. For this group, information on nationality and mental illness were also obtained: 195 were born in Asia, 23 in Europe and 8 in the United States. Finally, 205 reported not to have had a history of mental illness, whilst 9 said they had a history of mental illness and 12 preferred not to answer the question.

\section{Measures}

Divergent Thinking (DT; Guilford, 1967). Participants think of unusual uses for three inanimate objects, including brick, blanket, and wooden pencil. Participants have three minutes per object to write down as many uses as they can possibly think of. The number of responses was recorded as fluency score. Two independent judges, including one author of this study and a research colleague, rated each response's of originality on a scale from 1 to 6 ; inter-rater agreement was satisfactory with a correlation of .92 .

Runco Ideational Behaviour Scale (RIBS; Runco, Jonathan \& Lim, 2001). This self-report measure of creative ideation assesses an individual's use of, appreciation of and skill with ideas. Participants indicate on a five-point Likert scale the extent to which 23 statements describe their usual behavior; that is, overt actions and activities. Runco et al. (2001) reported satisfactory psychometric properties of the measure as well as discriminant validity but concluded that the scale would benefit from future refinements.

Biographical Inventory of Creative Behaviors (BICB; Batey, 2007). This is an assessment of spontaneous everyday creativity and creative achievement. Participants 
indicate, from a list of 34 activities, those in which they had been involved over the past 12 months. From this scale, one item was found contentwise to be inadequate and was excluded from all further analysis ${ }^{1}$.

NEO Five-Factor Inventory (FFI; Costa and McCrae, 1992). The 60-item scale is a short version of the NEO-PI-R and assesses the Big Five dimensions of personality on a five-point Likert scale. The Big Five personality factors are cross-culturally representative and show satisfactory reliability and validity (e.g. Costa \& McCrae, 1995; Goldberg \& Saucier, 1995). The current analysis only included the traits of Openness, Agreeableness and Conscientiousness.

Eysenck Personality Scale-Revised (EPQ-R; Eysenck, Eysenck \& Barrett, 1985). This short-version of the revised EPQ comprises 48 dichotomous (yes/ no) items that assess Extraversion, Neuroticism and Psychoticism, and also include a Lie scale. The EPQ has been demonstrated to have excellent reliability and validity (Eysenck et al., 1985).

Short-Scale Oxford-Liverpool Inventory of Feelings and Experiences (Short-Scale O-LIFE; Mason et al., 2005). This is a shortened version of the Oxford-Liverpool Inventory of Feelings and Experiences for schizotypy, containing 43 items for four subdimensions of unusual experiences (e.g. hallucinations and magical thinking), cognitive disorganisation (e.g. poor concentration and decision-making), introvertive ahedonia (e.g. avoidance of intimacy and lack of enjoyment), and impulsive non-conformity (e.g. antisocial behaviour and lack of self-control). Each sub-scale is assessed by 10 to 12 items.

Hypomania (Eckblad \& Chapman, 1986). It is a 48 items, non-timed questionnaire, which assesses the extent to which one's personality is overactive or

\footnotetext{
${ }^{1}$ The item read: Have you given anyone a present in the past 12 months?
} 
gregarious based on emotions, daily behaviours and activities. This scale was previously found to have satisfactory psychometric properties (Eckblad \& Chapman, 1986).

\section{Procedure}

BICB data was obtained through aggregating samples from several researchers²; some of them were collected in paper-and-pencil mode and some using online questionnaires. In all cases, the BICB was administered in a quiet setting and participants were free to take as much time as they pleased to complete the measure.

For a sub-sample, personality tests and additional creativity tests were administered in laboratory sessions with up to 15 participants at a time. The assessment booklet was organized in the order of measures as listed above. The divergent thinking tests were administrated and completed under strict timed conditions. Participants subsequently completed the remainder of the booklet at their own pace.

\section{Analysis}

Creative ideation was examined in an exploratory principal factor analysis following Kline's (1986) methods of test analysis. Subsequently, correlational analyses evaluated the association of creative ability, including divergent thinking originality and fluency, and factors of creative ideation with personality traits from the EPQ, the NEO, and the schizotypy scales, as well as hypomania.

\footnotetext{
${ }^{2}$ We thank Mark Batey for generously sharing his data on the BICB with us.
} 
Creative achievement was investigated in a latent class analysis using maximumlikelihood estimation with robust standard errors (MLR) in MPlus 4.1 (Muthén \& Muthén, 1998-2006). All BICB items were treated as categorical variables. To avoid local solutions on the likelihood surface (Hipp \& Bauer, 2006), 1000 early-stage and 100 finish-stage random starting values and several seed and perturbation values were generated to confirm that they converged to the same solution, in line with Silvia et al.'s (2009) analysis approach. Latent class models identify distinct groups or categories in data (Hagenaars \& McCutcheon, 2002) and assign each participant a probability of belonging to a given class. In this study, the choice of retained latent classes was based on entropy, likelihood ratio tests and information criteria, including Akaike's Information Criterion (AIC) and Bayesian Information Criterion (BIC; Muthén \& Muthén, 19982006). Furthermore, theoretical sensibility and parsimony were considered, preferring solutions with fewer classes of larger numbers. The latent class solution was further evaluated in a sub-sample. A multinomial regression model tested for the associations of creative ability and ideation, and personality with class membership. All dependent variables were $z$-transformed.

Results

Creative Ideation and Divergent Thinking

The initial communalities of two RIBS items were below recommended values of .25 (Kline, 1986); these items were excluded, as well as five items with extracted communalities below .25. An exploratory factor analysis of the remaining 16 items 
showed three factors with Eigenvalues of above 1 accounting for $54.95 \%$ of the total variance; a three-factor solution was also supported by the screeplot. Table 1 shows the factor loadings of the retained items on the three extracted dimensions after oblique rotation.

\section{Table 1}

The three ideation factors were readily identified as quantity of ideas $(n=9)$, absorption $(n=3)$ and originality $(n=5)$. The scales had coefficient alphas of $.88, .70$ and .73, respectively, and corresponding unit-weighted composite scores were calculated adjusting for the number of scale items.

In line with Silvia et al.’s (2008) findings on scoring divergent thinking tasks, fluency and originality scores of divergent thinking were added to build composite scores of creative fluency and originality, respectively. Table 2 shows the correlations between divergent thinking dimensions and factors of creative ideation. The coefficients were all positive and moderate, suggesting that creative ideation was distinct from creative ability. Divergent thinking dimensions of fluency and originality, however, were substantially correlated at .67.

Table $2 \& 3$ 


\section{Latent Class Analysis of Creative Achievement}

A three latent class solution proved the best fit to the data with the lowest BIC, AIC and LRT. Table 3 shows the average class-assignment probabilities. People, for whom class 1 was most likely, had a chance of $87 \%$ to be in class 1 and chances to be in one of the other two classes were $9 \%$ and $3.9 \%$; for class 2 , the probabilities were $91.1 \%$, and $8.1 \%$ and $0.8 \%$ for being in either of the other classes. Similarly, people for whom class 3 was most likely, had a $91.9 \%$ chance of being part of that class and chances to be else where were at $7.3 \%$ and $0.9 \%$. Overall, classes appeared to be well-separated. Figure 1 shows the three class profiles with the $\mathrm{x}$-axis reflecting the degree of creative achievement for a given creativity item, whose labels are shown along the y-axis. Class 3 included $15.9 \%(N=104)$ of the sample and hence, was the smallest identified group, which showed the highest creative achievement across items. The second-largest group included $36.7 \%(N=241)$ of the sample in class 1 . People in this class were on average quantitatively below the creative achievement of class 3 . However, they exceeded in event organization, management and joke invention. Also, this group showed a high probability for achievement in mentoring, leadership and interior design. Finally, class 2 was the largest group including $47.4 \%(N=311)$ of participants; it was clearly the least creative or creatively achieving group. Overall, classes appeared to differ to greater extent in quantity than in quality, suggesting that everyday creative achievement may be best understood as continuous distribution. 


\section{Personality and Creativity}

Table 4 shows Descriptives the EPQ and NEO personality traits, the schizotypy scales and hypomania and their correlations with divergent thinking and ideation controlling for age and sex.

Table 4

None of the EPQ scales was significantly correlated with creative ideation or divergent thinking. From the NEO, openness to experience associated with all three ideation dimensions with coefficients ranging from .18 to .46, and also shared a considerable amount of variance with creative ability of .30 and .28 for originality and fluency, respectively. Agreeableness was significantly, negatively correlated with the absorption factor of ideation. From the schizotypy scales, unusual experiences were positively related to quantity of ideas and originality, whereas cognitive disorganization was associated with absorption and impulsive non-conformity related to quantity of ideas and absorption; none of the schizotypy scales was associated divergent thinking. Hypomania was significantly and strongly correlated with all three factors of ideation but with not creative ability. In sum, openness was the only trait scale related to both creative ideation and creative ability but compared to all other personality traits, hypomania showed the most consistent associated with creative ideation. 
Table $5 \& 6$

To investigate the personality and creativity profile of the obtained latent classes of creative achievement, means were examined (Table 5), and a multinomial regression model tested for the effects of ideation, divergent thinking and personality traits on class membership (Table 6). The class of creative achievers scored higher than the other classes on factors of creative ideation, divergent thinking originality and fluency, Openness, Neuroticism, Extraversion, the schizotypy scales and hypomania; only in Agreeableness, creative achievers had a markedly lower mean score than classes of noncreative and medium achievers. The observed mean differences are in line with the expectations and lend tentative support for the validity of the accepted latent class solution.

In the multinomial regression model with the non-achieving class as reference group, one Standard Deviation (SD) increase in hypomania was significantly associated with a $76 \%$ increase in the odds of being in class 1 , which included people of medium creative achievement, and a $143 \%$ increase in the odds of being in class 3 , which was the group of most creative individuals. Also, one SD increase in Extraversion was significantly associated with an odds ratio of 2.64 of being in the (creative) class 3 compared to the non-creative group. No significant effects were observed for any of the other personality scales. 


\section{Discussion}

The aim of the current study was twofold: on one hand, components of creative competence were investigated upon their structure and inter-relations, and on the other creativity's relationship with personality traits was explored.

\section{Creative Ability, Ideation and Achievement}

A latent class analysis of spontaneous, everyday creative behaviors identified three groups of creative achievers, which partially confirmed Silvia et al.'s (2009) earlier findings. In line with their results, the largest recognized group represented individuals of no or very few creative achievements. A second group included people of medium creative achievements, who were also characterized by an enhanced tendency for creative achievement in interpersonal contexts, such as managing or mentoring people. The third class represented the smallest number of people, who were identified as high creative achievers across domains. The medium group overlapped with the high achieving class in domain of interpersonal accomplishments, but was overall more similar to the group of low creative achievement for the remaining domains. Overall, the classes' profiles were less clearly distinct than in Silvia et al.'s (2009) analysis, which is likely to be due to the differences in the employed measurement instruments of creative achievement. The currently investigated scale aims to assess spontaneous everyday creativity rather than exceptional creative accomplishments (cf. Batey, 2007). The results suggest that 
individuals differ quantitatively in everyday creative achievement, rather than qualitatively. It is possible that these quantitative differences become qualitative over time when individuals invest in and further develop their spontaneous creative activity. Such notions are presently speculative and future research is needed to examine developmental processes in creative achievement. It is clear, however, that researchers must carefully distinguish between everyday creative achievement and extraordinary creative accomplishments when investigating domain-specification versus generality.

The adequacy of the latent class solution was supported by mean differences in factors of creative ideation and divergent thinking, on which non-achievers and medium achievers with interpersonal competence were than high achievers. These differences, however, did not significantly contribute to predictions of latent class membership. That is, creative ability and ideation were found to be largely unrelated to actual creative achievement. Furthermore, divergent thinking fluency and originality were moderately and mostly non-significantly associated with factors of creative ideation. This is possibly due differences in the measurements' conceptualizations, whereby one taps maximum and the other one typical creative performance (Fiske \& Butler, 1963). Overall, psychometric instruments of distinct, but theoretically related components of creativity were found to be only loosely inter-linked. One hand, these results emphasize the complex, multifarious nature of creativity and creative competence (e.g. Mumford, 2003; Silvia et al., 2008), and stress on the other that any single psychometric test will inevitably fail to adequately operationalize creativity and its true scope. 
Latent classes of creative achievers differed in their mean personality scores with the creative group scoring higher on Openness, introvertive anhedonia, unusual experiences and hypomania, but lower on Agreeableness than their less creative counterparts. This is largely in line with previous research showing positive associations of Openness and schizotpy with creativity but negative links with Agreeableness (Batey \& Furnham, 2006; Feist, 1998); thus, the validity of the latent class solution was further supported. These mean differences did however not translate into significant predictor effects on creative achievement class membership. Only two personality dimensions Extraversion and hypomania - significantly contributed to the odds of being in the high creative achievement class compared to non- and/ or medium achievement groups. Extraversion was associated with being in the high achievement group but not the medium group, although both peaked in inter-personal creative accomplishments, such as managing and mentoring. Extraversion is known to predict social engagement, as well as managerial and leadership skills (e.g. Costa \& McCrae, 1992) and thus, heightened scores on this trait dimension are intuitive for the second and third class.

Hypomania clearly differentiated among the creative achievement classes with significant effects on the odds of being in the medium or high achievement class compared to the non-achieving group. Hypomania was also significantly correlated with factors of creative ideation, but showed no relationship with creative ability. Hypomania is characterized by flights of ideas, endless energy, euphoria and an excessive drive for success (Eckblad \& Chapman, 1986). Conceivably, these attributes are conducive for creative ideation (e.g. quantity of ideas) and creative achievement (i.e. high energy) but appear to be theoretically and empirically unrelated to creative ability. 
Openness to experience, which previous research identified as meaningful trait in creativity (e.g. Feist, 1998; Furnham et al., 2008), was significantly associated with divergent thinking fluency and originality and ideation factors of quantity and absorption. Openness shares variance with general intelligence (e.g. Ackerman \& Heggestad, 1997) and hence, the association with divergent thinking may be due to common characteristics of cognitive speed and flexibility. The lack of significant effects on latent classes of creative achievement, however, suggests that the role of Openness in creativity may have been previously overestimated.

\section{Conclusions}

This study highlights the multifarious nature of the construct creativity and its corresponding measures. Creative ideation was fairly independent of creative ability, and latent classes of creative achievement were not significantly associated to either ability or ideation. Without doubt, ability, ideation and achievement aspects are core components of creative competence; however, their complex inter-relations are yet to be fully disentangled. Furthermore, everyday creative achievements do largely form quantitative but less qualitative latent classes, and hypomania was shown to be a very promising candidate for future research studying the role of personality in creativity. The extreme diversity of creativity and its measures, however, evidently complicates a thorough understanding of personality's effects on this phenomenon. 


\section{References}

Ackerman, P. L. (1996). A theory of adult intellectual development: Process, personality, interests, and knowledge. Intelligence, 22, 227-257.

Ackerman, P. L., \& Heggestad, E. D. (1997). Intelligence, personality, and interests:

Evidence for overlapping traits. Psychological Bulletin, 121, 219-245.

American Psychiatric Association. (2000). Diagnostic and statistical manual of mental disorders. Fourth Edition. Text revision. Washington, D.C.: Author.

Barron, F. X. (1955). The disposition toward originality. Journal of Abnormal Social Psychology, 51, 478-485.

Barron, F. X., \& Harrington, D. M. (1981). Creativity, intelligence and personality. Annual Review of Psychology, 32, 439-476.

Batey, M. (2007). A psychometric investigation of everyday creativity. Unpublished Doctoral Thesis. University of London.

Batey, M., \& Furnham, A. (2008). The relationship between creativity, schizotypy and intelligence. Personality and Individual Differences, 45, 816-821.

Batey, M., \& Furnham, A. (2006). Creativity, intelligence and personality: A critical review of the scattered literature. Genetic, General and Social Psychology Monographs, 132, 355-429.

Claridge, G., McCreery, C., Mason, O., Bentall, R., Boyle, G., \& Slade, P. (1996). The factor structure of schizotypal traits: A large replication study. British Journal of Clinical Psychology, 35, 103-115.

Eckbald, M., \& Chapman, L. J. (1986). Development and validation of a scale for hypomanic personality. Journal of Abnormal Psychology, 95, 214-222. 
Eysenck, H. J. (1993). Creativity and personality: Suggestions for a theory. Psychological Inquiry, 4, 147-178.

Feist, G. J. (1998). A meta-analysis of the impact of personality on scientific and artistic creativity. Personality and Social Psychological Review, 2, 290-309.

Feist, G. J., \& Barron, F. X. (2003). Predicting creativity from early to late adulthood: Intellect, potential, and personality. Journal of Research in Personality, 37, 62-88.

Fiske, D. W., \& Butler, J. M. (1963). The experimental conditions for measuring individual differences. Educational and Psychological Measurement, 23, 249266.

Furnham, A., Batey, M., Anand, K., \& Manfield, J. (2008). Personality, hypomania, intelligence and creativity. Personality and Individual Differences, 44, 10601069.

Guilford, J. P. (1950). Creativity. American Psychologist, 5, 444-454.

Hargreaves, H. L. (1927). The "faculty" of imagination: An enquiry concerning the existence of a general "faculty," or group factor, of imagination. British Journal of Psychology Monograph Supplement, 3,1-74.

Hu, L., \& Bentler, P. M. (1999). Cut-off criteria for fit indexes in covariance structure analysis: Conventional criteria versus new alternatives. Structural Equation Modeling, 6, 1-55.

Kline, P. (1986). A handbook of test construction: Introduction to psychometric design. New York, US: Methuen. 
MacKinnon, D. W. (1962). The nature and nurture of creative talent. American Psychologist, 17, 484-495.

Mason, O., Claridge, G., \& Jackson, M. (1995). New scales for the assessment of schizotypy. Personality and Individual Differences, 1, 7-13.

Mumford, M. D. (2003). Where have we been, where are we going? Taking stock in creativity research. Creativity Research Journal, 15, 107-120.

Muthén, L.K., \& Muthén, B. O. (1998-2006). Mplus User's Guide. Forth Edition. Los Angeles, CA: Muthén \& Muthén.

Plucker, J. A., Runco, M. A. \& Lim, W. (2006). Predicting ideational behavior from divergent thinking and discretionary time on task. Creativity Research Journal, 18, 55-63.

Prentky, R. A. (2001). Mental illness and roots of genius. Creativity Research Journal, $13,95-104$.

Runco, M. A., Plucker, J. A., \& Lim, W. (2001). Development and psychometric integrity of a measure of ideational behavior. Creativity Research Journal, 13, 393-400.

Simonton, D. K. (1976). Biographical determinants of achieved eminence: A multivariate approach to the Cox data. Journal of Personality and Social Psychology, 35, 218-226.

Silvia, P. J., Nusbaum, E. C., Berg, C., Martin, C., \& O'Conner, A. (2009). Openness to experience, plasticity, and creativity: Exploring lower-order, higher-order, and interactive effects. Journal of Research in Personality, 43, 1087-1090. 
Silvia, P .J., Kaufman, J. C., \& Pretz, J . E. (2009). Is creativity domain-specific? Latent class models of creative accomplishments and creative self-descriptions. Psychology of Aesthetics, Creativity and the Arts, 3, 139-148.

Silvia, P. J., Winterstein, B. P., Willse, J. T., Barona, C. M., Cram, J. T., Hess, K. I., Martinez, J. L., \& Richard, C. A. (2008). Assessing creativity with divergent thinking tasks: Exploring the reliability and validity of new subjective scoring methods. Psychology of Aesthetics, Creativity, and the Arts, 2, 68-85.

Sternberg, R. J., \& Lubart, T. I. (1991). An investment theory of creativity and its development. Human Development, 34, 1-32.

Taylor, C. W. (1988). Various approaches to and definitions of creativity. The nature of creativity: Contemporary psychological perspectives. London, UK: Cambridge University Press. 


\section{Figure Captions}

Figure 1

Profiles of a three class solution for creative achievement.

Note. Items are shown along the $\mathrm{x}$-axis; probabilities of achievement are shown on the y-axis. 
Tables

Table 1

Pattern matrix of factor solution of RIBS under oblique rotation.

\begin{tabular}{|c|c|c|c|c|}
\hline & & \multicolumn{3}{|c|}{ Factor } \\
\hline & & 1 & 2 & 3 \\
\hline RIBS 2 & Think often about ideas & .801 & -.009 & -.035 \\
\hline RIBS 3 & Excited by own ideas & .704 & .086 & -.132 \\
\hline RIBS 1 & Many wild ideas & .681 & .099 & -.035 \\
\hline RIBS 5 & Ideas no one else has & .655 & -.072 & .168 \\
\hline RIBS 6 & Play with ideas & .638 & .058 & -.015 \\
\hline RIBS 9 & Active thinker & .594 & .014 & .202 \\
\hline RIBS 4 & Lots of ideas and solutions & .589 & -.077 & .166 \\
\hline RIBS 7 & Important to think bizarre & .576 & .055 & .001 \\
\hline RIBS 8 & Able to generate ideas & .557 & -.066 & .182 \\
\hline RIBS 17 & Often let mind wonder & .072 & .705 & .017 \\
\hline RIBS 18 & Seen as absent-mined & -.087 & .620 & .126 \\
\hline RIBS 16 & No exclusive focus & .187 & .590 & -.051 \\
\hline RIBS 20 & Create new solutions & -.081 & .078 & .780 \\
\hline RIBS 23 & Inventions and improvements & .082 & -.011 & .562 \\
\hline RIBS 21 & Uniquely combining ideas & .234 & -.011 & .513 \\
\hline RIBS 22 & Recognized as great thinker & .210 & -.066 & .507 \\
\hline RIBS 19 & Thinking things through & -.010 & .199 & .432 \\
\hline
\end{tabular}

Note. Factor loadings above .30 are shown in bold. $N=223$, after listwise omission. 
Table 2

Correlations of divergent thinking, i.e. fluency and originality, with factors of creative ideation.

\begin{tabular}{llcccc}
\hline & & 1 & 2 & 3 & 4 \\
\hline 1 & Quantity & - & & & \\
2 & Absorption & .36 & - & & \\
3 & Originality & .59 & .32 & - & \\
4 & DT Originality & .22 & .10 & .10 & - \\
5 & DT Fluency & .28 & .11 & .12 & .67 \\
\hline
\end{tabular}

Note. Correlations of .22 and above are significant at $\mathrm{p}>.01$. Key: DT $=$ Divergent Thinking. 
Table 3

Average latent class probabilities for most likely latent class membership (rows) by latent class (columns).

\begin{tabular}{lccc}
\hline & Class 1 & Class 2 & Class 3 \\
\hline Class 1 & .870 & .090 & .039 \\
Class 2 & .081 & .911 & .008 \\
Class 3 & .073 & .009 & .919 \\
\hline
\end{tabular}

Note. This class solution is depicted in Figure 1; the entropy value was .772. 
Table 4

Correlations between measures of creativity and personality scales.

\begin{tabular}{llllccccc}
\hline & & \multicolumn{1}{c}{ Ideation } & \multicolumn{3}{c}{$D T$} \\
& $M$ & $S D$ & $\alpha$ & Quan & Abs & Orig & DTO & DTF \\
\hline Psychoticism & 3.27 & 1.90 & .46 & .13 & .11 & .12 & .01 & .10 \\
Neuroticism & 5.71 & 3.46 & .82 & .13 & .22 & -.01 & .05 & .03 \\
Extraversion & 7.19 & 2.70 & .68 & .15 & .02 & .11 & .11 & .15 \\
Openness & 26.07 & 4.66 & .50 &. $\mathbf{4 6}$ & $\mathbf{. 1 9}$ & .18 &. $\mathbf{3 0}$ &. $\mathbf{2 8}$ \\
Agreeableness & 27.25 & 5.29 & .65 & -.18 & $\mathbf{- 2 1}$ & -.17 & .03 & .05 \\
Conscientiousness & 27.37 & 5.75 & .74 & .02 & -.13 & .20 & -.03 & -.04 \\
Unusual Experiences & 4.88 & 2.89 & .71 &. $\mathbf{2 6}$ & .12 &. $\mathbf{2 5}$ & .14 & .06 \\
Cognitive Disorganization & 5.21 & 2.76 & .72 & .07 & $\mathbf{. 2 3}$ & -.10 & .11 & .11 \\
Introvert Anhedonia & 3.53 & 2.04 & .54 & .01 & .12 & .13 & .03 & .03 \\
Impulsive Non-Conformity & 3.87 & 2.11 & .48 & $\mathbf{. 2 5}$ & $\mathbf{. 1 9}$ & .17 & .16 & .17 \\
Hypomania & 19.58 & 7.51 & .82 & $\mathbf{. 4 3}$ & $\mathbf{. 3 2}$ & $\mathbf{. 3 0}$ & .05 & .07 \\
\hline
\end{tabular}

Note. All coefficients are corrected for age and sex $(N=185$, after listwise omission). Coefficients of .19 and above are significant at $p<.01$, two-tailed, and are shown in bold. 
Table 5

Means and Standard Deviations (SD) across latent classes of creative achievement for creative ideation, divergent thinking and personality scales.

\begin{tabular}{lcccccc}
\hline & \multicolumn{2}{c}{ Non-creative } & \multicolumn{2}{c}{ Medium } & \multicolumn{2}{c}{ Creative } \\
\hline Quantity & 3.04 & $(0.76)$ & 3.44 & $(0.58)$ & 3.34 & $(0.71)$ \\
Absorption & 2.64 & $(0.81)$ & 2.74 & $(0.89)$ & 3.01 & $(0.82)$ \\
Originality & 2.99 & $(0.71)$ & 3.23 & $(0.62)$ & 3.26 & $(0.74)$ \\
DT Originality & 8.97 & $(3.23)$ & 9.12 & $(2.82)$ & 10.00 & $(3.53)$ \\
DT Fluency & 13.62 & $(7.79)$ & 14.98 & $(7.14)$ & 16.28 & $(8.16)$ \\
Openness & 25.67 & $(4.93)$ & 26.12 & $(3.98)$ & 27.23 & $(5.47)$ \\
Agreeableness & 27.43 & $(4.62)$ & 27.40 & $(5.95)$ & 26.26 & $(5.37)$ \\
Conscientiousness & 27.78 & $(5.15)$ & 26.93 & $(6.48)$ & 27.31 & $(5.49)$ \\
Psychoticsim & 3.28 & $(1.83)$ & 3.16 & $(2.02)$ & 3.56 & $(1.81)$ \\
Neuroticism & 5.58 & $(3.23)$ & 5.74 & $(3.69)$ & 6.00 & $(3.58)$ \\
Extraversion & 6.35 & $(2.55)$ & 7.81 & $(2.60)$ & 8.16 & $(2.74)$ \\
Cognitive Disorganization & 5.20 & $(2.78)$ & 5.22 & $(2.76)$ & 5.23 & $(2.78)$ \\
Introvert Anhedonia & 3.74 & $(2.04)$ & 3.15 & $(1.90)$ & 3.94 & $(2.30)$ \\
Impulsive Non-conformity & 3.74 & $(1.93)$ & 3.65 & $(2.19)$ & 4.88 & $(2.20)$ \\
Unusual Experiences & 4.56 & $(2.82)$ & 4.90 & $(3.07)$ & 5.90 & $(2.34)$ \\
Hypomania & 16.91 & $(6.68)$ & 21.02 & $(7.29)$ & 23.69 & $(7.69)$ \\
\hline & & & & & &
\end{tabular}


Table 5

Multinomial regression Odds Ratios (OR) with Confidence Intervals of 95\% (CI $95 \%)$

\begin{tabular}{|c|c|c|c|c|c|c|}
\hline \multirow[b]{3}{*}{ Quantity } & \multicolumn{3}{|c|}{ Class $1(N=77)$} & \multicolumn{3}{|c|}{ Class $3(N=29)$} \\
\hline & \multirow{2}{*}{$\begin{array}{c}\text { OR } \\
1.36\end{array}$} & \multicolumn{2}{|c|}{$(C I 95 \%)$} & \multirow{2}{*}{$\begin{array}{c}O R \\
0.58\end{array}$} & \multicolumn{2}{|c|}{$(C I 95 \%)$} \\
\hline & & $(0.81$, & 2.28) & & $(0.27$ & 1.26) \\
\hline Absorption & 1.00 & $(0.67$ & $1.50)$ & 1.74 & $(0.95$ & $3.21)$ \\
\hline Originality & 1.14 & $(0.71$, & 1.82) & 0.72 & $(0.35$ & 1.47) \\
\hline DT Originality & 0.74 & $(0.45$ & $1.21)$ & 0.90 & $(0.43$ & 1.88) \\
\hline DT Fluency & 1.26 & $(0.77$ & 2.07) & 1.50 & $(0.73$ & $3.10)$ \\
\hline Openness & 0.88 & $(0.58$ & $1.35)$ & 1.37 & $(0.77$ & 2.46) \\
\hline Agreeableness & 1.23 & $(0.81$, & 1.88) & 1.02 & $(0.54$ & 1.93) \\
\hline Conscientiousness & 1.01 & $(0.67$ & $1.53)$ & 1.57 & $(0.84$ & 2.96) \\
\hline Psychoticsim & 1.04 & $(0.70$ & $1.56)$ & 0.93 & $(0.51$ & $1.72)$ \\
\hline Neuroticism & 1.20 & $(0.75$ & 1.94) & 0.78 & $(0.39$ & $1.56)$ \\
\hline Extraversion & 1.39 & $(0.88$ & $2.20)$ & 2.64 & (1.26, & 5.52) \\
\hline Cognitive Disorganization & 1.00 & $(0.63$ & $1.58)$ & 0.62 & $(0.32$ & $1.20)$ \\
\hline Introvert Anhedonia & 0.88 & $(0.54$ & $1.42)$ & 1.61 & $(0.83$ & $3.10)$ \\
\hline Impulsive Non-conformity & 0.74 & $(0.46$ & $1.20)$ & 1.39 & $(0.70$ & 2.74) \\
\hline Unusual Experiences & 1.04 & $(0.68$ & 1.61) & 1.67 & $(0.88$ & $3.16)$ \\
\hline Hypomania & 1.76 & (1.04, & 2.97) & 2.43 & (1.14, & 5.16) \\
\hline
\end{tabular}

Note. Regression models were adjusted for sex and age; reference group was Class $2(N=84)$. Significant ORs are shown in bold. $N=190$ after listwise omission. 
Figure 1

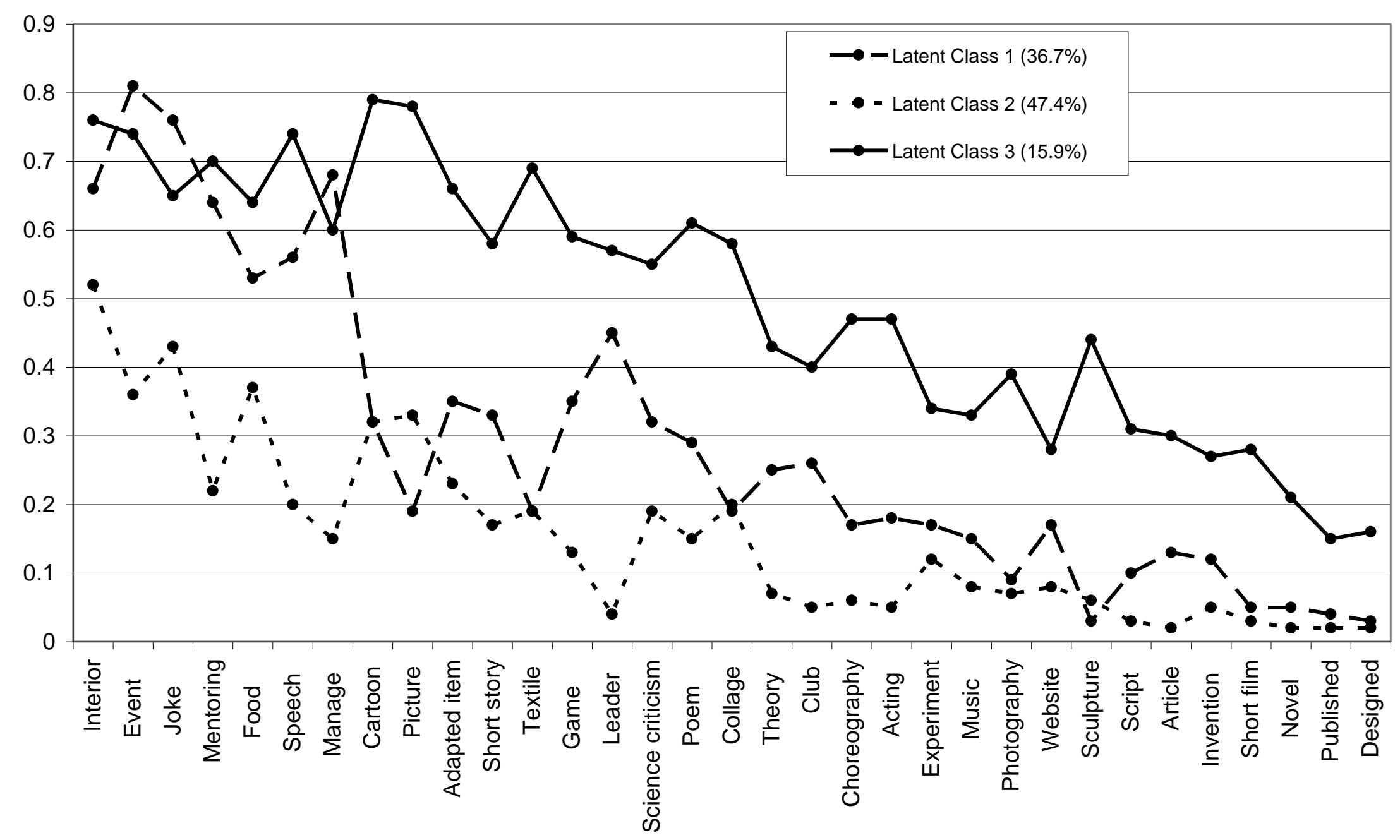


